\title{
First-principle calculations of effective mass of silicon crystal with vacancy defects
}

\author{
ShuYing Zhong ${ }^{1,2, *}$, Musheng Wu ${ }^{1}$, XUEling LeI $^{1}$ \\ ${ }^{1}$ Institute of Physics and Communication \& Electronics, Jiangxi Normal University, Nanchang 330022, P.R.China \\ ${ }^{2}$ School of Materials Science and Engineering, Nanchang University, Nanchang 330031, P.R.China
}

The energy band structures and electron (hole) effective masses of perfect crystalline silicon and silicon with various vacancy defects are investigated by using the plane-wave pseudopotential method based on density functional theory. Our results show that the effect of monovacancy and divacancy on the energy band structure of crystalline silicon is primarily reflected in producing the gap states and the local states in valence band maximum. It also causes breaking the symmetry of energy bands resulting from the Jahn-Teller effect, while only producing the gap states for the crystalline silicon with hexavacancy ring. However, vacancy point defects could not essentially affect the effective masses that are derived from the native energy bands of crystalline silicon, except for the production of defect states. Simultaneously, the Jahn-Teller distortions only affect the gap states and the local states in valence band maximum, but do not change the symmetry of conduction band minimum and the nonlocal states in valence band maximum, thus the symmetry of the effective masses. In addition, we study the electron (hole) effective masses for the gap states and the local states in valence band maximum.

Keywords: effective mass; vacancy; silicon; first-principles calculations

(C) Wroclaw University of Technology.

\section{Introduction}

Silicon, as the most important industrial material, has been widely used in various devices, such as advanced electronic devices [1], power devices [2], solar cells [3, 4] and microelectronic systems [5]. Due to limitations of the fabrication process, the ideal silicon crystal cannot be obtained in practice because of a large number of defects caused by the process of ion implantation, radiation or mechanical erosion. The presence of defects would bring the additional defect states, which directly influences the optoelectronic properties of materials [6].

Considering the significant effect of defects on devices and crystal quality, the defects arising during the process of crystal growth have been extensively studied in last decades, mostly focusing on the diffusion of the intrinsic point defects [7]. It is well known that in crystalline silicon, a vacancy defect is a kind of important intrinsic point defect.

*E-mail: shuyingzhong@ 163.com
So far, lots of reports have been related with the mechanical and electronic properties of the vacancy defects in crystalline silicon, which mainly referred to the defects of monovacancy, divacancy and hexavacancy [8-12]. Generally, the simplest monovacancy plays a key role in self-diffusion and impurity diffusion. Compared with other vacancies, divacancy has attracted extensive attention from both theoretical and experimental aspects as it is easy to be formed during the irradiation process, and thus is stable. For example, the structure and formation energy of the charged state of the divacancy defect have been studied experimentally $[13,14]$ and using theoretical first-principles calculations [15-18]. Additionally, hexavacancy, as the smallest ring vacancy, can be regarded as an effective adsorption center for hydrogen, oxygen and transition metal atom [19].

As we know, the defects in crystal often mix with various other defects instead of appearing as single one. The effect of an individual kind of vacancy defect on the electrical properties of crystal is difficult to be measured only by experiment. 
Therefore, theoretical research becomes important because it can investigate one kind of defect independently. Moreover, conductivity, which is the principal physical quantity to describe the electronic properties of materials, is in proportion to carrier mobility and inversely proportional to effective mass of electron or hole. Obviously, the effective mass of electron or hole is closely related to the conductive properties of crystalline silicon. Up to now, there has only been few literature that reported the effect of the vacancy defects on the effective masses derived from energy band. Motivated by this, in this work the effective masses of electron or hole in crystalline silicon with various kinds of vacancy defects (monovacancy, divacancy and hexavacancy) are systematically studied by using first-principles calculations. Then, the influence of the type of defect on the active mass is discussed.

\section{Model and methodology}

\subsection{Theoretical model}

Crystalline silicon adopts the structure of diamond with $8 \mathrm{Si}$ atoms in unit cell. The supercell containing $216 \mathrm{Si}$ atoms with $3 \times 3 \times 3$ model is used to produce defects in crystal so that the interaction between the defect and its image could be neglected. The crystalline structure with monovacancy, divacancy and hexavacancy ring defects can be obtained by removing one, two or six $\mathrm{Si}$ atoms from the supercell, respectively. The optimized configuration with the lowest energy is selected to be further studied.

\subsection{Methodology}

All calculations are performed by using the VASP [20, 21] (Vienna ab initio Simulation Package) within the projector augmented-wave (PAW) approach [22]. The ground state of the electronic structure is described within density functional theory (DFT) using the generalized gradient approximation (GGA) with Perdew-Wang (PW91) exchange correlation function [23]. The energy cutoff for expansion of wave functions and potentials is $300 \mathrm{eV}$. Monkhorst-Pack special k-point method is used with a grid of $2 \times 2 \times 2$ [24]. The entire systems are relaxed by conjugate gradient method until the force on each atom is less than $0.01 \mathrm{eV}$.

\section{Results and discussions}

\subsection{Geometry optimization}

Firstly, the geometry of silicon crystal with a defect is optimized. The relaxed structures are shown in Fig. 1. The distances between $\mathrm{Si}-\mathrm{Si}$ atoms $\left(\mathrm{d}_{\mathrm{Si}-\mathrm{Si}}\right)$ around the monovacancy defect are all equal to $3.86 \AA$ before relaxation. After relaxation, four distances between $\mathrm{Si}-\mathrm{Si}$ atoms decrease to $3.53 \AA$, while the other two decrease to $3.03 \AA$. Obviously, the structure around the monovacancy defect distorts. It is typically called as the JahnTeller effect which influences the electronic properties of silicon crystal with monovacancy defect. This is in agreement with the previous report [25]. With respect to divacancy defect, it is found that the optimized structure relates to the initial structure. By fine tuning the positions of six $\mathrm{Si}$ atoms around the divacancy defect, two different configurations with almost degeneration energy are obtained finally. The difference lies in the six $\mathrm{Si}-\mathrm{Si}$ distances that are formed between two $\mathrm{Si}$ atoms in every three $\mathrm{Si}$ atoms around the defect. For the first configuration, four $\mathrm{Si}-\mathrm{Si}$ distances $\mathrm{d}_{\mathrm{Si}-\mathrm{Si}}$ are $3.49 \AA$, while the other two are $2.80 \AA$, as shown in Fig. 1b, which is named as LP divacancy defect. However, for the second configuration, four $\mathrm{Si}-\mathrm{Si}$ distances $\mathrm{d}_{\mathrm{Si}-\mathrm{Si}}$ are $3.10 \AA$, and the other two are $3.62 \AA$, as shown in Fig. 1c, which is named as RB divacancy defect. From the structure point of view, local distortion could be observed for the two configurations with divacancy defects, and thus lower the structural symmetry. This indicates the appearance of the Jahn-Teller effect. From the energy point of view, the total energy of the silicon crystal with LP divacancy defect is only by $3 \mathrm{meV}$ lower than that with RB divacancy defect, in accord with the reports by Pesola et al. [10] and Makhov et al. [26]. Therefore, the small energy difference shows that the two configurations could both exist in realistic conditions. With regard to hexavacancy ring defect, the $\mathrm{Si}-\mathrm{Si}$ distances around the defect all decrease from $3.84 \AA$ to $2.74 \AA$ after relaxation, as shown in Fig. 1d. 


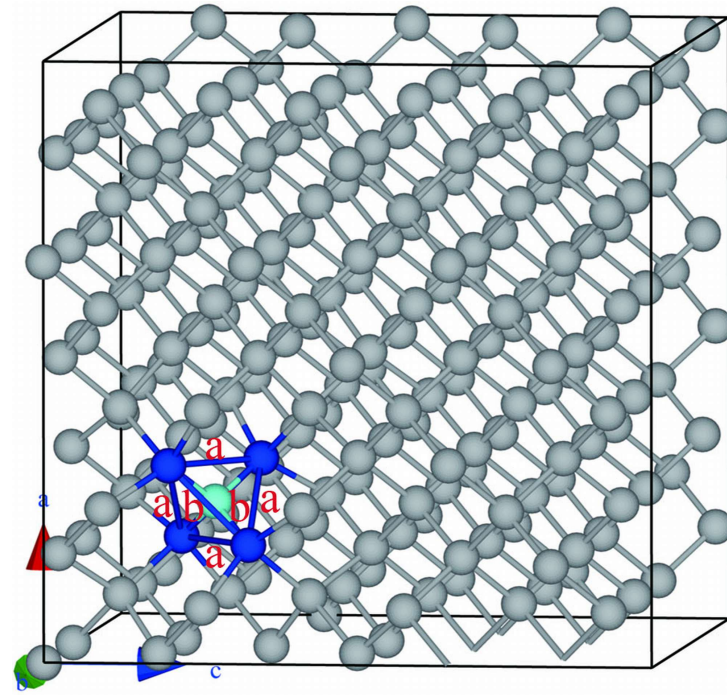

(a)

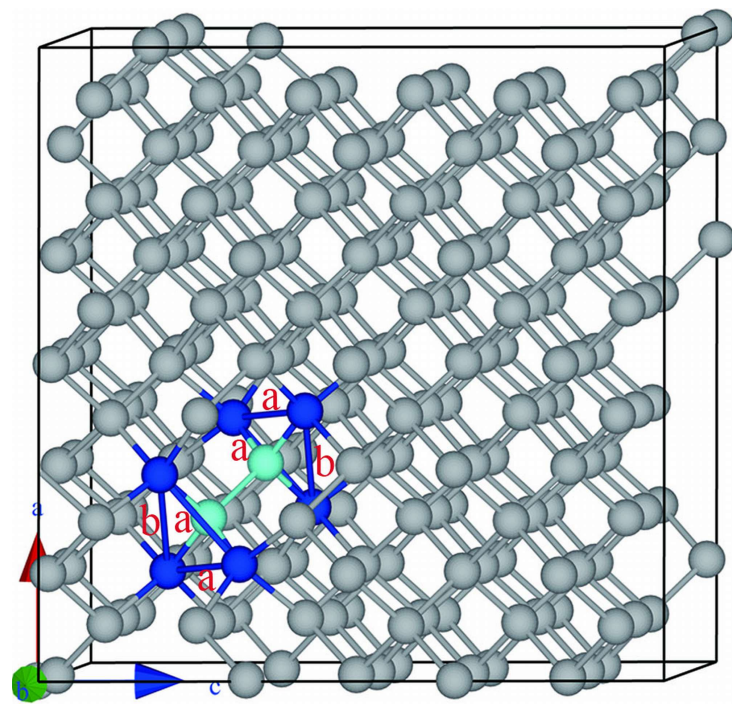

(c)

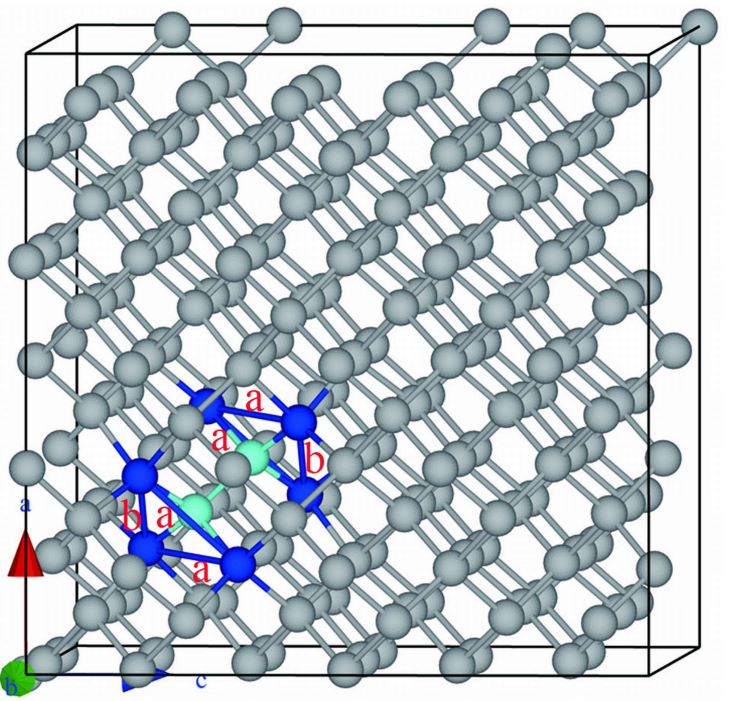

(b)

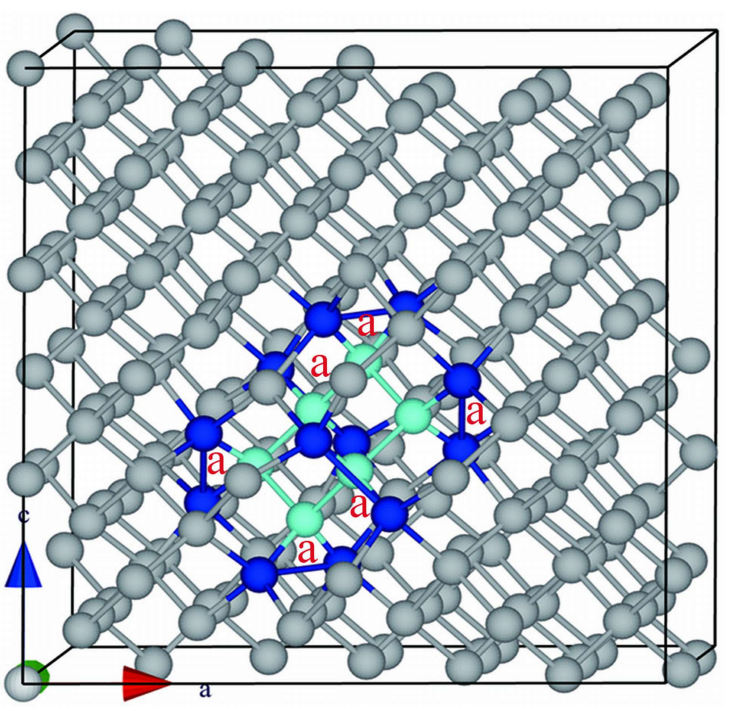

(d)

Fig. 1. Schematic view of crystalline silicon with monovacancy (V1), divacancy(V2) and hexavacancy (V6) defects (The light blue small balls denote the vacancy sites, the dark blue small balls denote the nearest neighbor atoms to the vacancy sites); (a) monovacancy defect; (b) LP divacancy defect; (c) RB divacancy defect; (d) hexavacancy defect.

\subsection{Energy band structures}

Next, we will investigate the electronic structures of perfect silicon and silicon with defects. The band structure of the perfect silicon crystal is shown in Fig. 2 (Fermi level is set to be $0 \mathrm{eV}$, and the same hereinafter). The high symmetry kpoints of Brillouin zone along the band paths in
Fig. 2 are $\mathrm{G}(0,0,0), \mathrm{F}(0,0.5,0), \mathrm{Q}(0,0.5,0.5)$ and $\mathrm{Z}(0,0,0.5)$. The results clearly show that silicon crystal is a semiconductor with indirect band gap. The valence band maximum (VBM) locates at $G$ point, while conduction band minimum (CBM) locates at $\mathrm{F}$ point. The band that includes the $\mathrm{CBM}$ is drawn with blue solid rhombus, which is marked with band "1". Another band that includes 
the VBM is drawn with red hollow rhombus, which is marked with band "2". The band "2" is twofold degenerate because the spin-orbital coupling is not considered in our calculations. Moreover, it is evidently found that the energy bands along G-F-Q and G-Z-Q pathways are symmetrical due to the cubic symmetry in a perfect silicon crystal.

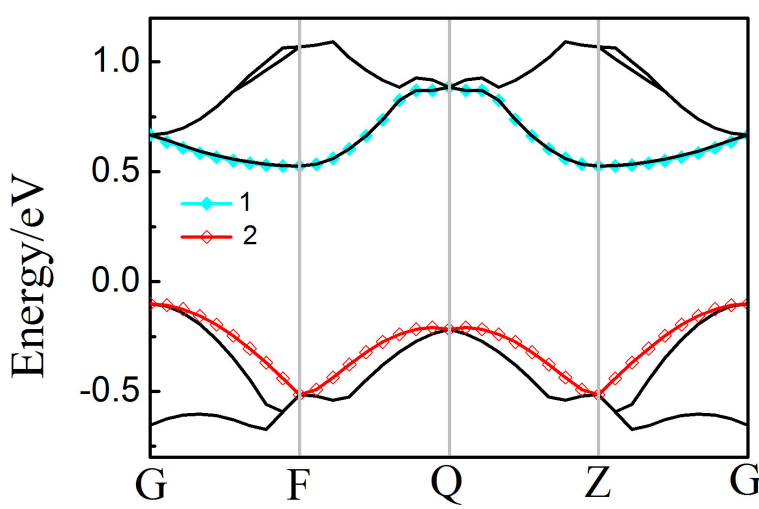

Fig. 2. Energy band structure of a perfect silicon crystal.

The band structure of silicon crystal with monovacancy defect is shown in Fig. 3. It is found that the band including VBM (band "2") exhibits the characteristics of localized state and the corresponding charge density mainly concentrates around the vacancy defect by band decomposed charge density analysis. In contrast to the band structure of perfect crystal, two evident defect bands stand above the Fermi level in the crystalline silicon with monovacancy defect, which are marked with band "3" (the curve drawn with purple hollow triangle) and band "4" (green solid triangle). The two defect bands are also related with the localized states and have the asymmetric characteristics. The bands along G-F-Q and G-Z-Q pathways and the band degeneracy are quite different. It indicates that the localized cubic symmetry has changed. Combined the localized structure with monovacancy defect, it can be understood that the change of localized symmetry results from the Jahn-Teller distortion.

The band structures with divacancy defects are shown in Fig. 4 and Fig. 5. Similar to the case of monovacancy defect, the band with VBM

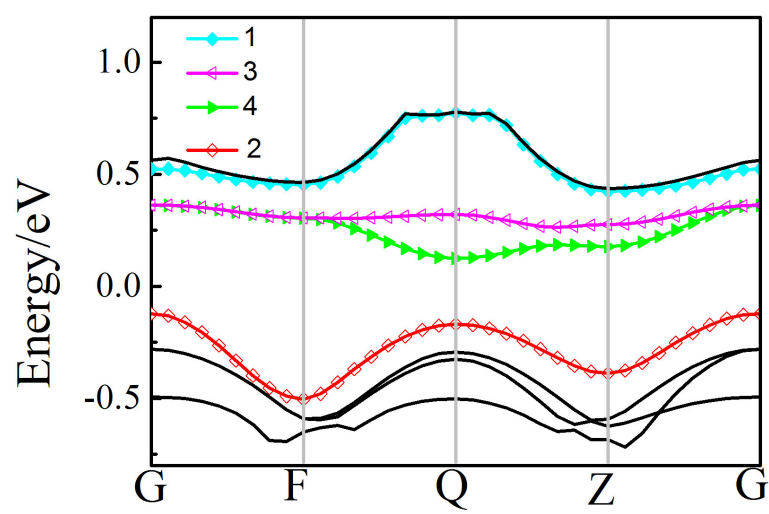

Fig. 3. Energy band structure of a silicon crystal with monovacancy defect.

(band "2") corresponds to the localized state, and two clear defect bands (band "3" and "4") occur above the Fermi level. The dispersion of the two bands relates to their pathways, and shows the asymmetric character along G-F-Q and G-Z-Q pathways. Similar to the case of monovacancy, the reason results from the Jahn-Teller distortion, regardless of LP divacancy defect or RB divacancy defect, thus lowering the original cubic symmetry.

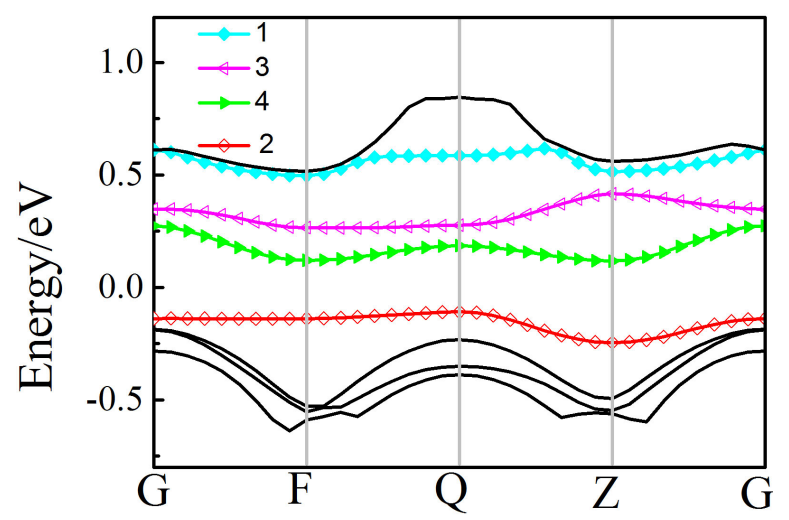

Fig. 4. Energy band structure of a silicon crystal with LP divacancy defects.

Fig. 6 shows the band structure of silicon crystal with hexavacancy defect. It is different from the cases of monovacancy and divacancy defects. Firstly, the band with VBM corresponds to extended state instead of localized state by band decomposed charge density analysis. Secondly, the defect bands (band "3" and "4") above the Fermi 


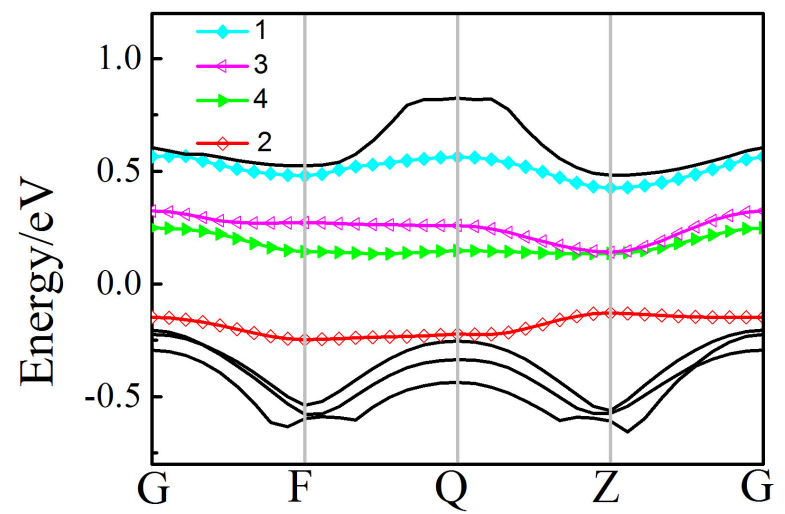

Fig. 5. Energy band structure of a silicon crystal with $\mathrm{RB}$ divacancy defects.

level are very close to conduction band. Finally, the band structure exhibits high symmetry. In other words, the bands along G-F-Q and G-Z-Q pathways are symmetrical. Moreover, it is worthwhile to note that the Jahn-Teller distortion is not found in crystalline silicon with hexavacancy defect. Therefore, it can be confirmed that the asymmetry of band relates to the Jahn-Teller distortion in the silicon crystals with monovacancy and divacancy defects.

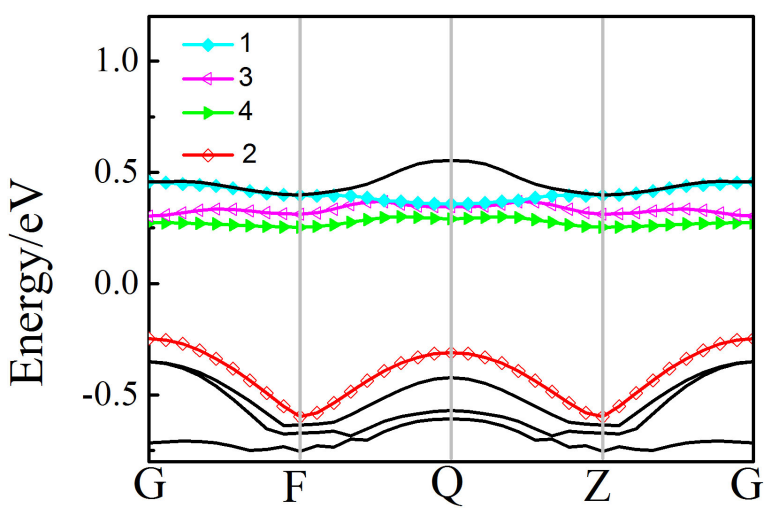

Fig. 6. Energy band structure of a silicon crystal with hexavacancy defects.

\subsection{Effective mass of electron and hole}

As silicon is an important material in solar cells and electronic devices, the electronic conductivity is very important to its realistic applications. The theory of effective mass can be regarded as a direct method to understand the electronic properties of a material. As a consequence, the related calculations of effective mass for silicon crystal with vacancy defect can be used to study the influence of defects on its conductivity. The effective mass of an electron or a hole can be obtained by the following formula [27, 28]:

$$
m_{e(\hbar)}^{*}=h^{2}\left(\frac{d^{2} E(k)}{d k^{2}}\right)^{-1}
$$

where $\bar{h}$ is reduced Planck's constant, $\mathrm{k}$ is wave vector, $\mathrm{E}(\mathrm{k})$ is electronic energy for the wave vector.

The effective mass of electron near the CBM is considered firstly. It should be noted that the bottom of conduction band in our calculations is the lowest unoccupied band, which does not include the defect states, corresponding to the band " 1 " in all of the band structures. The calculated effective masses are shown in Table 1. For a perfect silicon crystal, the effective mass at $F$ point strongly depends on the direction. The effective mass along FQ direction is $0.197 \mathrm{~m}_{0}$ ( $\mathrm{m}_{0}$ is free electron mass), which is much smaller than the effective mass of $0.95 \mathrm{~m}_{0}$ along FG direction, in agreement with the results reported by Wang et al. [29]. Moreover, the effective mass at $F$ point $(0,0.5,0)$ is equal to that at $\mathrm{Z}$ point $(0,0,0.5)$. It means that the effective masses at $\mathrm{F}$ point along $\mathrm{FQ}$ and $\mathrm{FG}$ directions are equal to each other, as well as at $\mathrm{Z}$ point along $Z Q$ and $Z G$ directions. It originates from the symmetry of the bands along G-F-Q and G-Z-Q pathways, showing the characteristics of cubic symmetry in a perfect silicon crystal. From Table 1, it can be seen that the electron effective masses at point $F$ and point $Z$ are almost equivalent when vacancy defects exist in crystalline silicon. Specially, the effective mass of electron at point $\mathrm{F}$ slightly increases along FG direction when the monovacancy defect and LP divacancy defect are induced, while it is almost unchanged along FQ direction. On the contrary, for the silicon crystal with RB divacancy defect and hexavacancy defect, the effective mass of electron at point $\mathrm{F}$ along $\mathrm{FG}$ direction decreases a little, whereas it increases along FQ direction. Particularly, the effective mass of electron increases greatly from $0.197 \mathrm{~m}_{0}$ in the perfect 
Table 1. The calculated electron effective masses for the bottom of the conduction bands (band " 1 " shown in all energy band structures) in crystalline silicon with or without the vacancy defects.

\begin{tabular}{lcccc}
\hline & $\begin{array}{c}\mathrm{m}_{\mathrm{e}}^{*} / \mathrm{m}_{0} \\
{[\mathrm{FG}]}\end{array}$ & $\begin{array}{c}\mathrm{m}_{\mathrm{e}}^{*} / \mathrm{m}_{0} \\
{[\mathrm{FQ}]}\end{array}$ & $\begin{array}{c}\mathrm{m}_{\mathrm{e}}^{*} / \mathrm{m}_{0} \\
{[\mathrm{ZQ}]}\end{array}$ & $\begin{array}{c}\mathrm{m}_{\mathrm{e}}^{*} / \mathrm{m}_{0} \\
{[\mathrm{ZG}]}\end{array}$ \\
\hline \hline Perfect crystal & 0.950 & 0.197 & 0.197 & 0.950 \\
Monovacancy & 1.174 & 0.190 & 0.199 & 1.066 \\
LP divacancy & 1.013 & 0.230 & 0.183 & 1.182 \\
RB divacancy & 0.813 & 0.269 & 0.277 & 0.572 \\
Hexavacancy & 0.805 & 1.236 & 1.467 & 0.800 \\
\hline
\end{tabular}

crystal to $1.236 \mathrm{~m}_{0}$ in the crystal with hexavacancy defect.

Next, the effective masses of a hole near the VBM are calculated and listed in Table 2. Since the bands containing VBM for the crystal with monovacancy and divacancy defects are apparently localized, the hole effective masses calculations are carried out for the bands with non-localized states. Therefore, the band " 2 " is used to calculate the hole effective masses in the perfect crystal and the crystal with hexavacancy defect, while the first band related to non-localized state under the band "2" is used in the crystal with monovacancy and divacancy defects. For the bands that are considered, however, only the effective masses of holes at $\mathrm{G}$ and Q points are calculated because the tops of the band locate at $\mathrm{G}$ and $\mathrm{Q}$ points. For a perfect crystal, the effective masses at $G$ point along GF and GZ directions are equivalent, as well as the effective masses at $\mathrm{Q}$ point along $\mathrm{QF}$ and $\mathrm{QZ}$ directions. It shows that the effective masses at $\mathrm{G}$ and $\mathrm{Q}$ points along two different pathways are symmetrical and the difference between them is very small. When vacancy defects are introduced, the symmetry of effective mass of holes retains and the values are somewhat larger than those in a perfect crystal.

The effective mass of an electron or a hole of the defect bands and the localized bands near the VBM in a crystal with various kinds of vacancy defects are shown in Table 3 . The positive value corresponds to the effective mass of an electron while the negative value corresponds to the effective mass
Table 2. The calculated hole effective masses for the top of the valence bands (the band related to the non-localized states) in crystalline silicon with or without the vacancy defects.

\begin{tabular}{lcccc}
\hline & $\begin{array}{c}\mathrm{m}_{\mathrm{h}}^{*} / \mathrm{m}_{0} \\
{[\mathrm{GF}]}\end{array}$ & $\begin{array}{c}\mathrm{m}_{\mathrm{h}}^{*} / \mathrm{m}_{0} \\
{[\mathrm{GZ}]}\end{array}$ & $\begin{array}{c}\mathrm{m}_{\mathrm{h}}^{*} / \mathrm{m}_{0} \\
{[\mathrm{QZ}]}\end{array}$ & $\begin{array}{c}\mathrm{m}_{\mathrm{h}}^{*} / \mathrm{m}_{0} \\
{[\mathrm{QF}]}\end{array}$ \\
\hline \hline Perfect crystal & 0.278 & 0.278 & 0.233 & 0.233 \\
Monovacancy & 0.357 & 0.356 & 0.270 & 0.230 \\
LP divacancy & 0.318 & 0.336 & 0.300 & 0.305 \\
RB divacancy & 0.232 & 0.308 & 0.366 & 0.389 \\
Hexavacancy & 0.329 & 0.325 & 0.284 & 0.284 \\
\hline
\end{tabular}

of a hole. The effective mass of an electron is only calculated with respect to the unoccupied defect bands. In contrast, the effective masses of holes are calculated at the tops of localized bands near the VBM. The results for monovacancy defect show that the effective masses of holes near the top of band are somewhat smaller. For instance, the effective masses of holes at point $\mathrm{G}$ along GF and GZ directions are $0.180 \mathrm{~m}_{0}$ and $0.311 \mathrm{~m}_{0}$, respectively. Similarly, the effective masses of electron at point $\mathrm{Q}$ along QF and QZ, corresponding to the bottom of interval band " 4 ", are $0.338 \mathrm{~m}_{0}$ and $0.542 \mathrm{~m}_{0}$. For the crystal with divacancy, regardless of LP or $\mathrm{RB}$, the effective masses of holes at VBM strongly depend on directions. For example, the effective masses of holes at point $\mathrm{G}$ along QF and QZ directions in the crystal with LP divacancy defect are $1.593 \mathrm{~m}_{0}$ and $0.429 \mathrm{~m}_{0}$, respectively. Likewise, the effective masses of holes at point $F$ along FG and FQ directions in the crystal with LP divacancy defect are $0.447 \mathrm{~m}_{0}$ and $0.999 \mathrm{~m}_{0}$, and $0.841 \mathrm{~m}_{0}$ and $0.378 \mathrm{~m}_{0}$ at point $\mathrm{Z}$ along $\mathrm{ZQ}$ and $\mathrm{ZG}$ directions, respectively. Compared with the crystal with LP divacancy defect, the difference is more obvious in the crystal with RB divacancy defect. The effective masses of holes at point $\mathrm{Z}$ along $\mathrm{ZQ}$ and $\mathrm{ZG}$ directions are $9.982 \mathrm{~m}_{0}$ and $0.479 \mathrm{~m}_{0}$, respectively. For the crystal with hexavacancy defect, either defect band " 3 " or band " 4 " have many valleys. Furthermore, the energies for these two bands are very close to each other. Therefore, the calculated values of electron effective mass are very abundant. It can be seen from the calculated results that the effective 
Table 3. The calculated electron (hole) effective masses for the defect states and the bands near the top of the valence band (the non-localized states) in crystalline silicon with the vacancy defects (negative values refer to hole effective masses, and the positive ones to electron effective masses).

\begin{tabular}{|c|c|c|c|c|c|c|c|c|c|}
\hline & \multirow{3}{*}{ band } & \multicolumn{8}{|c|}{$\mathrm{m}^{*} / \mathrm{m}_{0}$} \\
\hline & & \multicolumn{2}{|c|}{ G } & \multicolumn{2}{|c|}{$\mathrm{F}$} & \multicolumn{2}{|c|}{ Q } & \multicolumn{2}{|c|}{$\mathrm{Z}$} \\
\hline & & GF & GZ & FG & FQ & QF & QZ & ZQ & ZG \\
\hline \multirow{3}{*}{ Mono } & 3 & & & 0.877 & & & & & 0.633 \\
\hline & 4 & & & 0.876 & & 0.338 & 0.542 & 1.220 & 0.334 \\
\hline & 2 & -0.180 & -0.311 & & & -0.281 & -0.363 & & \\
\hline \multirow{3}{*}{ LP } & 3 & & 1.054 & 0.583 & & & 0.538 & & \\
\hline & 4 & & & 0.447 & 0.999 & & & 0.841 & 0.378 \\
\hline & 2 & -43.787 & -0.576 & & & -1.593 & -0.429 & & \\
\hline \multirow{3}{*}{$\mathrm{RB}$} & 3 & & & & & 10.311 & & 0.508 & 0.296 \\
\hline & 4 & & & 0.404 & & & & 9.982 & 0.479 \\
\hline & 2 & -0.760 & & & & -0.728 & -1.878 & & \\
\hline \multirow{2}{*}{ Hexa } & 3 & 0.523 & 0.514 & 0.953 & 0.326 & 4.707 & 4.720 & 0.290 & 0.849 \\
\hline & 4 & & & 2.274 & 0.661 & 0.813 & 0.813 & 0.611 & 2.395 \\
\hline
\end{tabular}

masses have apparently symmetric characteristics with respect to $\mathrm{Q}$ point. It further shows that the band of the crystal with hexavacancy is symmetric, in accord with the previous energy band analysis.

\section{Conclusions}

The band structures and effective mass of electron (hole) in silicon crystal with various kinds of vacancies are studied by using first-principles methods. Our results show that:

(1) The band structures in perfect crystalline silicon and in silicon crystal with hexavacancy defect have cubic symmetry. However, the systems with monovacancy and divacancy defects exhibit typically asymmetric characteristics due to the presence of defect bands caused by the Jahn-Teller distortion.

(2) The effective mass of electron that corresponds to the intrinsic band in perfect crystalline silicon has high symmetry. The property of effective mass of electron in crystal with vacancy defect is similar to that in perfect system except for the larger effective mass in crystal with hexavacancy defect. The effective mass of hole that corresponds to the intrinsic band in perfect crystalline silicon also has high symmetry, and the values for the high symmetry points are almost equivalent. Moreover, the values and symmetry of the hole effective masses are basically kept in the crystals with vacancy defects, implying that the non-localized band symmetry of VBM and CBM in monovacancy and divacancy defects is not changed by Jahn-Teller distortion.

(3) The effective masses of electron (hole) for the localized state bands at VBM and defect states in the crystal with monovacancy defect are not large and exhibit directivity. The effective masses of electron (hole) that correspond to the localized bands in the crystals with LP and RB divacancy defects also exhibit apparent directivity. On the other hand, the corresponding effective masses of electron (hole) in the crystal with hexavacancy defect exhibit clear symmetry. It shows that the localized state bands at VBM and defect states in the crystals with monovacancy and divacancy defects can be affected by the Jahn-Teller distortion.

(4) By analyzing the influence of vacancy defects on the band structures and the effective masses, and further investigating the effect of vacancy defects on conductivity and carrier mobility in crystal, the intrinsic relationship between vacancy defects and the electronic properties of crystalline silicon, has been relealed. 


\section{Acknowledgements}

This work was supported by the National Science Foundation of China under the Grant No. 51361022 and the Science Foundation of Jiangxi normal University under the Grant No. 12017174.

\section{References}

[1] Rong H.S., LiU A.S., Richard J., Oded C., Dani H., Remus N., Alexander F., Mario P., $\mathrm{Na}$ ture, 433 (2005), 292.

[2] Dominique S., Roland E., Sol. Energ. Mat. Sol. C., 72 (2002), 27.

[3] Liang L., XU Q.F., Hu M.L., Sun H., Xiang G.H., Zhou L.B., Acta Phys. Sin.-Ch. Ed., 62 (2013), 037301-1.

[4] Bruton T.M., Sol. Energ. Mat. Sol. C, 72 (2002), 3.

[5] Dosunmu O.I., Cannon D.D., Emsley M.K., GHYSElEN B., LiU J.F., Kimerling L.C., UNLU M.S., IEEE J. Sel. Top. Quant., 10 (2004), 694.

[6] Santos P., Coutinho J., Rayson M.J., Briddon P.R., Phys. Status Solidi C, 9 (2012), 2000.

[7] Kamiyama E., Sueoka K., Vanhellemont J., $J$. Appl. Phys., 111 (2012), 083507.

[8] Centoni S.A., SAdigh B., Gilmer G.H., Lenosky T.J., Tomásdíaz D.L.R., Musgrave C.B., Phys. Rev. B, 72 (2005), 195206.

[9] Mellouhi F.E., Mousseau N., Ordejón P., Phys. Rev. B, 70 (2004), 205202.

[10] Pesola M., Boehm J., Pöykкö S., Nieminen R.M., Phys. Rev. B, 58 (1998), 1106.

[11] Wright A.F., Phys. Rev. B, 74 (2006), 165116.

[12] Hasings J.L., Estreicher S.K., Fedders P.A., Phys. Rev. B, 56 (1997), 10215.

[13] Watkins G.D., Corbett J.W., Phys. Rev., 138 (1965), A543.

[14] Nagai Y., Inoue K., Tang Z., Yonenaga I., Chiba T., Saito M., Hasegawa M., Physica B, $340-342$ (2003), 518.
[15] Iwata J.I., Shiraishi K., Oshiyama A., Phys. Rev. $B, 77$ (2008), 115208.

[16] Ogut S., Chelikowsky J.R., Phys. Rev. Lett., 83 (1999), 3852.

[17] Ogut S., Chelikowsky J.R., Phys. Rev. B, 64 (2001), 245206.

[18] Wixom R.R., Wright A.F., Phys. Rev. B, 74 (2006), 205208.

[19] Estreicher S.K., Hastings J.L., Fedders P.A., Appl. Phys. Lett., 70 (1997), 432.

[20] Kresse G., Hafner J., Phys. Rev. B, 47 (1993), 558.

[21] Kresse G., Furthmuller J., Comp. Mater. Sci., 6 (1996), 15.

[22] Perdew J.P., Chevary J.A., Vosko S.H., Jackson K.A., Pederson M.R., Singh D.J., Fiolhais C., Phys. Rev. B, 46 (1992), 6671.

[23] Kresse G., Joubert D., Phys. Rev. B, 59 (1999), 1758.

[24] Monkhorst H.J., PaCK J.D., Phys. Rev. B, 13 (1976), 5188.

[25] Puska M. J., Poźkkö S., Pesola M., Nieminen R.M., Phys. Rev. B, 58 (1998), 1318.

[26] Makhov D.V., Lewis L.J., Phys. Rev. B, 72 (2005), 073306.

[27] Wang Y.Z., XU Z.P., Zhang W.X., Zhang X. Wang Q., Zhang L., Acta Phys. Sin.-Ch. Ed., 63 (2014), 237101.

[28] Zheng S.W., Fan G.H., He M., Zhao L.Z., Acta Phys. Sin.-Ch. Ed., 63 (2014), 057102.

[29] Wang C.S., Klein B.M., Phys. Rev. B, 24 (1981), 3393. 